\title{
Impurity Effects and Spin Polarizations in a Narrow Quantum Hall System
}

\author{
Tapash Chakraborty* \\ Max-Planck Institut für Physik Komplexer Systeme, Nöthnitzer Straße 38, D-01187 Dresden, Germany
}

\author{
K. Niemelä and P. Pietiläinen \\ Theoretical Physics, University of Oulu, Linnanmaa, FIN-90570 Oulu, Finland
}

(June 26, 2021)

\begin{abstract}
The temperature dependence of electron spin polarization for a narrow quantum Hall system shows behavior analogous to that of a two-dimensional system at major filling factors. At the lowest half-filled quantum Hall state for which no two-dimensional analog exists, we find a stable spin partially-polarized system. Periodic Gaussian repulsive impurities (antidots) in such a system leads to novel spin transitions at $\nu=\frac{1}{3}$ and $\nu=\frac{1}{2}$ and the pair-correlation functions provide clues about nature of different ground states in the system. These results can be explored in optical spectroscopy and optically pumped NMR Knight shift measurements.
\end{abstract}

One of the perhaps most spectacular demonstration of electron correlations in nature is the fractional quantum Hall effect (FQHE) [1] for which an almost complete picture of the electronic properties at $1 / m$ filling of the lowest Landau level, $m$ being an odd integer, is available [2]. During the rapid developments of our understanding of the effect that ensued [3], one of the fundamental properties of the system became well established, i.e., that spin degree of freedom plays a very important role in the ground state and elementary excitations in the FQHE systems [4,5]. In fact, among the many theoretical predictions made within the framework of the incompressible fluid state, only a few have received direct experimental support as yet and those include effects based on spin polarizations of the two-dimensional electron system (2DES) in the FQHE regime [5, 6]. Temperature dependence of the spin polarization, calculated recently for the FQHE states and predicted to have a non-monotonical behavior for the spin-singlet ground states [7], has also received experimental support [8].

When the lowest Landau level is completely filled, the ground state is fully spin polarized due to electronelectron interactions [9]. In recent experiments on spin polarizations at and around $\nu=1$, a precipitous fall in the spin polarization was observed when either one moves slightly away from $\nu=1$ or the temperature is increased at $\nu=1$ 10] (exceptions also exist, see e.g., Ref. [11.8 where no such drop of spin polarization at $\nu \approx 1$, or at $\nu \approx \frac{1}{3}$ was observed). In this paper we investigate the spin polarizations of electrons in a narrow quantum Hall wire. We find that most of the features observed earlier in two dimensions are preserved in a narrow channel. We also demonstrate that the presence of a periodic array of Gaussian scatterers (antidot model) has remarkable effects on spin polarizations of the incompressible states in a quantum wire. We find abrupt change in spin polarizations for a given filling factor as the impurity strength is increased. In addition, the pair-correlation functions provide a glimpse of the nature of different ground states in the system.

In our model for the QHE in a narrow channel, we consider a finite number of spin polarized electrons interacting via the long-range Coulomb potential [12] and confined by a potential which is parabolic [13] in one direction and flat in the other. A strong magnetic field is applied perpendicular to the $x y$ plane. The electrons are confined in a cell of length $a$ in $x$ direction and the width of the cell depends on the strength of the confining potential $\left(\frac{1}{2} m^{*} \omega_{0}^{2} y^{2}\right)$ relative to the strength of the interactions and also on the length of the cell. We impose a periodicity condition in the $x$ direction. For example, we use antiperiodic boundary conditions for $4 n$ electrons so that the non-interacting ground states have zero total momentum [12].

Electrons are assumed to occupy only the lowest Landau level due to the strong magnetic field. The effective magnetic length in the problem is $\lambda=\left(\hbar / m^{*} \Omega\right)^{\frac{1}{2}}$, where $m^{*}$ is the electron effective mass, $\Omega=\sqrt{\omega_{0}^{2}+\omega_{c}^{2}}$ and $\omega_{c}=e B / m^{*}$ is the cyclotron frequency. The singleelectron wave functions are plane waves in $x$-direction and oscillator wave functions in $y$-direction centered at $y_{0}=2 \pi \lambda^{2} m /\left(a \sqrt{1+\left(\omega_{0} / \omega_{c}\right)^{2}}\right)$. Here $m$ is the momentum quantum number. The corresponding energies, excluding the constant Landau level energy are: $E=(2 \pi)^{2}(\lambda / a)^{2} m$ in units of $E_{0}=\left(\hbar^{2} / 2 m^{*} \lambda^{2}\right)\left(\omega_{0}^{2} / \Omega^{2}\right)$. The Hamiltonian in the lowest Landau level, which includes contributions from the electron-electron interactions and the neutralizing background, is numerically diagonalized for a few-electron system with spin degree of freedom properly included. A phase diagram is then obtained by plotting the energy gap (energy separation between the translationally invariant ground state and the lowest excited state) [12] for various values of $a$ and the increasing strength of the interaction $\mathcal{E}_{c}=e^{2} / \epsilon \lambda$ with respect to the energy unit $E_{0}$. We should point out that evaluation of filling factors in a 1D system is somewhat tricky. Here the single-particle states corresponding to a particular Landau "level" are not degenerate. One way 
is to calculate the areal electron density and number of fluxes through a unit area and determine $\nu$ as the ratio of these two quantities. Alternatively, we count the number of occupied states and divide the number of electrons by that. Both methods are somewhat arbitrary: one has to choose properly either the width of the density profile in the first case (we have used full width at half maximum) or, in the second approach, which state should be considered as occupied. We have checked that both methods agree reasonably well. The $\frac{1}{3} \mathrm{FQH}$ state in the present system is also identified from the momentum distribution function $\langle n(k)\rangle=\left\langle 0\left|a_{k}^{\dagger} a_{k}\right| 0\right\rangle$ by comparing it with that for a Laughlin-like wave function.

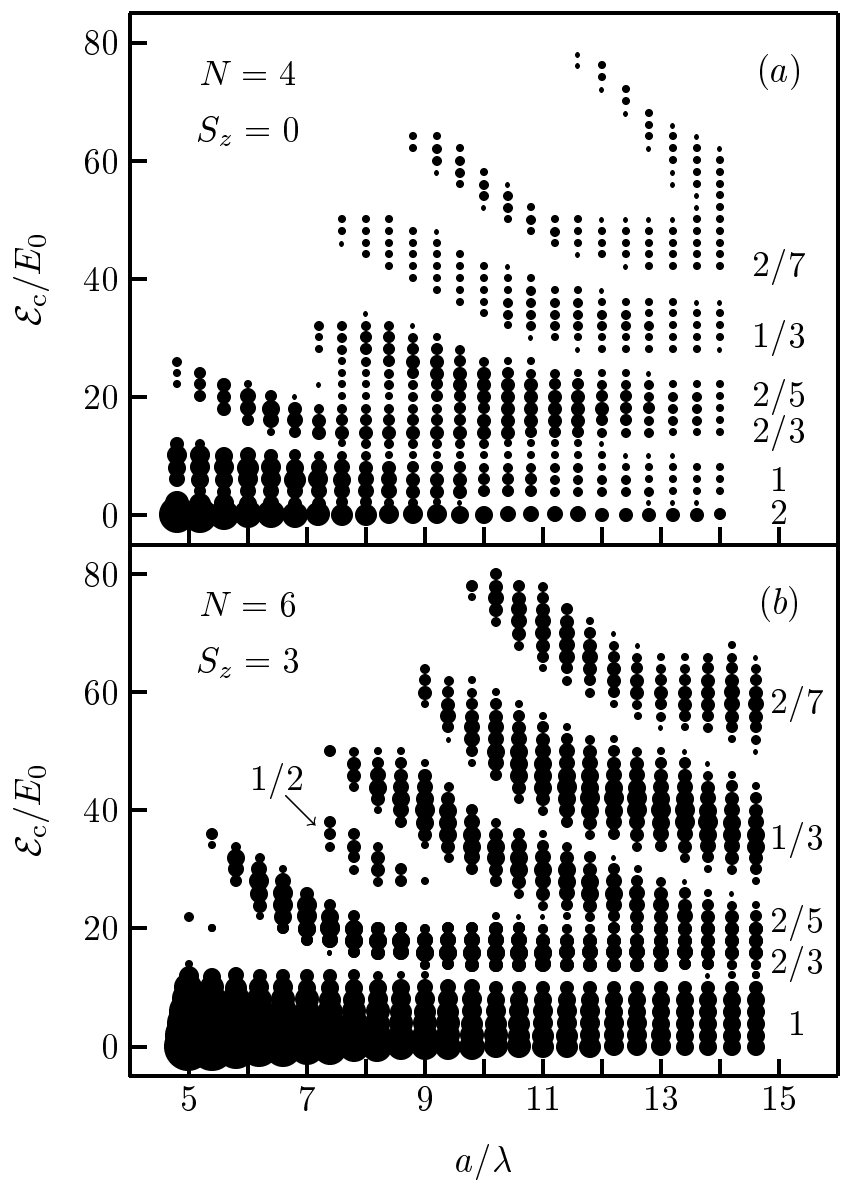

FIG. 1: Phase diagram for electrons in a impurity-free narrow channel quantum Hall system, (a) with and (b) without spin degree of freedom included.

In Fig. 1, we present the results for the phase diagram, calculated for a (a) system of six spinless electrons and (b) a system of four electrons with $S_{z}=0$ (Zeeman energy not included) and for $\alpha=\omega_{0} / \omega_{c}=0.23$ which is appropriate for $B=10 \mathrm{~T}$ and $\hbar \omega_{0}=4 \mathrm{meV}$. The area of a filled dot is directly proportional to the energy gap. As is evident in the figure, several quantum Hall states are stable with large energy gaps in the parameter range considered in this work. For the $N=4$ system the $\nu=\frac{1}{2}$ state, though supposed to exist, cannot be resolved in this phase diagram. In Fig. 1(a), the $\nu=\frac{1}{2}$ states are expected to lie between $\nu=\frac{2}{3}$ and $\nu=\frac{2}{5}$. In general, the energy gaps are larger for spinless electrons [Fig. 1(b)] because in the other case there are low-lying spin excitations available.

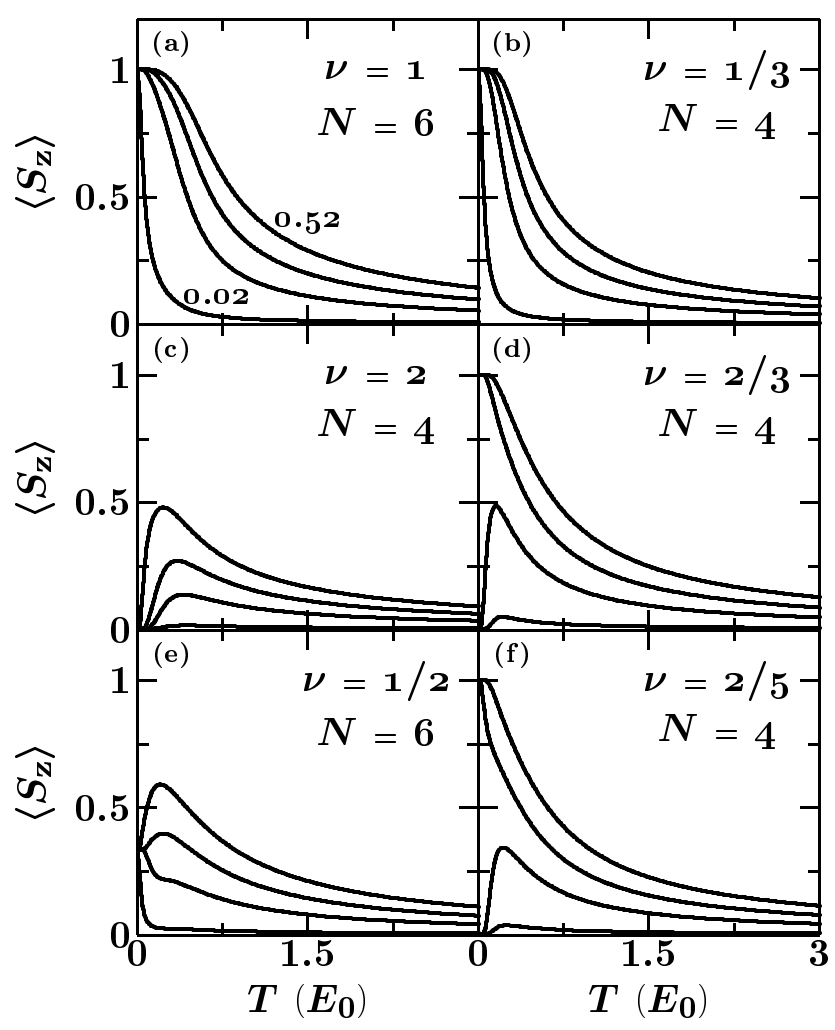

FIG. 2: Spin polarization $\left\langle S_{z}\right\rangle$ vs $T$ for $\nu=1,2, \frac{2}{3}, \frac{1}{2}, \frac{1}{3}$ and $\frac{2}{5}$ for an impurity-free system.

The temperature dependence of spin polarization for various filling factors found in the phase diagram is calculated by a method we developed earlier [7]. For the $\nu=\frac{1}{2}$ results we have employed a six electron system. The spin polarization $\left\langle S_{z}(T)\right\rangle$ is calculated from

$$
\left\langle S_{z}(T)\right\rangle \equiv \frac{1}{Z} \sum \mathrm{e}^{-\varepsilon_{j} / k T}\left\langle j\left|S_{z}\right| j\right\rangle
$$

where $Z=\sum_{j} \mathrm{e}^{-\beta \varepsilon_{j}}$ is the canonical partition function and the summation is over all states including all possible polarizations. Here $\varepsilon_{j}$ is the energy of the state $|j\rangle$ with Zeeman coupling included. A direct measurement of 
$\left\langle S_{z}(T)\right\rangle$ is possible through the NMR Knight-shift measurements and also via optical spectroscopic measurements [10]. These experiments provide a unique probe of spin polarizations in the system.

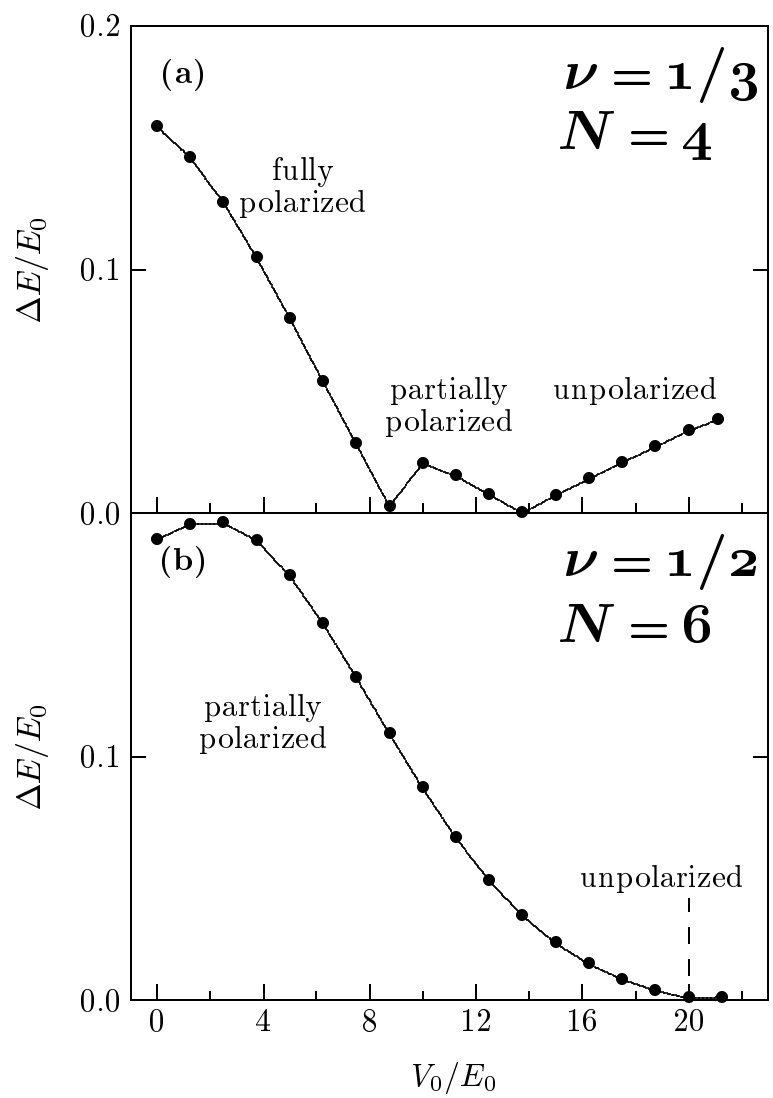

FIG. 3: Energy gap at (a) $\nu=\frac{1}{3}$ and (b) $\nu=\frac{1}{2}$ in a narrow channel quantum Hall system as a function of the strength of the Gaussian repulsive scatterer at the origin and $d=1$.

Our results for $\left\langle S_{z}(T)\right\rangle$ vs $T$ at $\nu=1,2, \frac{2}{3}, \frac{2}{5}, \frac{1}{3}$ and $\frac{1}{2}$ are shown in Fig. 2. In these calculations the magnetic field was kept fixed at $10 \mathrm{~T}$ and the $g$-factor is varied $(0.02-0.52)$. At $\nu=1$, we find the results to be similar to those for the two-dimensional systems [7] and the system is fully spin polarized even for very low values of the Zeeman energy. Qualitatively similar behavior is also seen at $\nu=\frac{1}{3}$. In the same way, $\nu=2$ is a spin-unpolarized state even at the highest value of the Zeeman energy considered and $\nu=\frac{2}{3}$ and $\nu=\frac{2}{5}$ are spin-unpolarized states at low Zeeman energies with a non-monotonic temperature dependence as predicted for a 2DES [7]. Such a non-monotonical behavior is observed in experiments on a 2DES [8]. Clearly, the correspondence with the spin polarization in a two dimensional system gives us confidence that our classification of the QH states in a narrow channel system is essentially correct. At $\nu=\frac{1}{2}$ we find a spin partially-polarized state.

A two-dimensional electron gas with a periodic array of scatterers (antidots) is an attractive system to look for the signature of a fermi surface around $\nu=\frac{1}{2}$ [14, 15 where the well known commensurability resonances 16] are exploited. In a $2 \mathrm{D}$ quantum Hall system, even the innocuous $\frac{1}{3}$ state is known to change its spin polarization in the presence of antidot potentials [17]. We have studied the electronic properties of a quantum wire when there is a Gaussian scatterer of the form

$$
V^{\mathrm{imp}}(\mathbf{r})=V_{0} e^{-(\mathbf{r}-\mathbf{R})^{2} / d^{2}}
$$

in the cell which, as a result of the boundary conditions, represents a periodic array of scatterers. Here $V_{0}$ is the potential strength, $d$ is the width of the potential (in units of magnetic length) and $\mathbf{R}$ is the impurity position within the cell.

In Fig. 3, we present the energy gap (without including the Zeeman contribution) at (a) $\nu=\frac{1}{3}$ and (b) $\nu=\frac{1}{2}$ for electrons in a narrow channel as a function of the impurity potential strength. The energy gap decreases and finally vanishes when the impurity strength is increased. With further increase in strength of the impurity potential the gap however starts to reappear but with different spin polarizations (and non-FQH states, as discussed below). At $\nu=1 / 3$, an increase in the strength of the impurity potential seems to cause rapid transitions from a spin polarized state to a partially polarized state and finally to an unpolarized state. At $\nu=\frac{1}{2}$, the energy gap also drops rapidly and the spin state changes from the partially polarized to the spin unpolarized state, albeit with an extremely small energy gap. We should add a cautionary statement here about the $\nu=\frac{1}{2}$ results in the presence of a strong impurity potential: the system is too large to check the improvement achieved in convergence with respect to increase in basis states.

In order to identify the various phases seen at $\nu=\frac{1}{3}$ in Fig. 3, we have calculated the pair-correlation functions for a four-electron system in various situations as shown in Fig. 4. Figures 4(a) and 4(b) correspond to the FQH case in the absence of any antidot potential. Clearly, the extra structure in $g_{\uparrow \downarrow}(r)$ as compared to $g_{\uparrow \uparrow}(r)$ is due to the Pauli principle. Antidot potential has only minor effects in the distribution of electrons in the $\frac{1}{3}$-state as long as there is a non-vanishing energy gap in the excitation spectrum (Fig. 3). This is evident when one compares Fig. 4 (a),(b) and (c),(d): the antidot potential only slightly localizes the electrons. On the other hand, there is a dramatic change in the pair-correlation functions after the original FQH-gap has vanished i.e., $V_{0}=10$ [Fig. 4 (e) and 4(f)]. The electrons then are distributed very differently from $\nu=\frac{1}{3} \mathrm{FQH}$-state and 
are strongly localized along the $y$-axis depending upon the spin polarization of the electrons. For $V_{0} \gg 8$ the states are also non-FQH like.
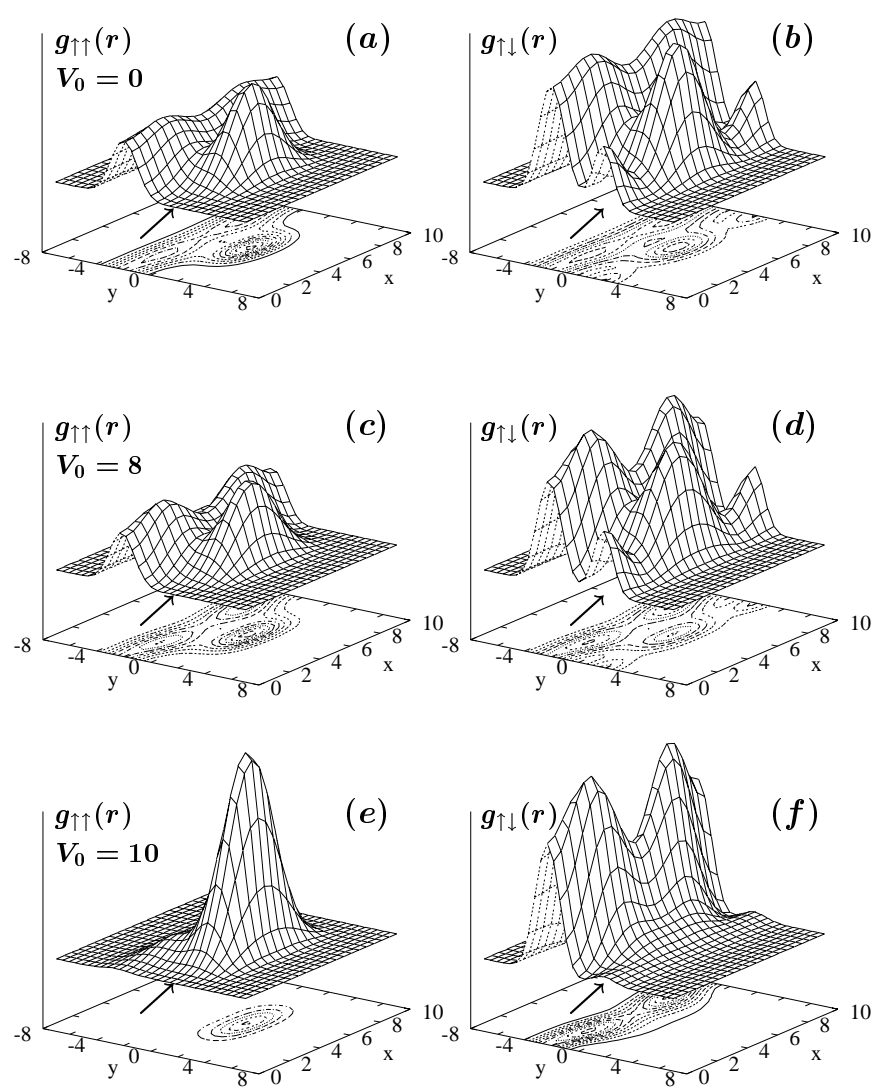

FIG. 4: Pair-correlation functions for the $\frac{1}{3}$-FQH state with $V_{0}=0$ (a) and (b) and $V_{0}=8$ (c) and (d). The non-FQH $\frac{1}{3}$-states are shown for $V_{0}=10(\mathrm{e})$ and $(\mathrm{f})$. The arrow indicates the position where one electron is kept fixed.

To summarize our results, we have studied the temperature dependence of the spin polarizations of interacting electrons in a narrow quantum Hall system. We find that there is a clear correspondence with the two-dimensional behavior at most of the major filling factors. At the half-filled Landau level we find a spin partially-polarized state. The $\frac{1}{3} \mathrm{FQH}$ state is found to be stable against the influence of the impurity potential until the energy gap vanishes. The system then goes to a non-FQH state and the impurity potential strongly localizes the electrons. While in transport measurements there are signatures of QH states at $\nu=\frac{1}{2}$ in a narrow channel 18], optical spectroscopy and optically pumped NMR Knight shift measurements are perhaps more suitable for observation of the temperature dependence of spin-polarization at $\nu=\frac{1}{2}$ and $\nu=\frac{1}{3}$ in a quantum wire as predicted here.

One of us (TC) would like to thank Peter Fulde for his kind hospitality. KN is supported by the grant of Jenny and Antti Wihuri's foundation.

* On leave from: Institute of Mathematical Sciences, Taramani, Madras 600 113, India

[1] D.C. Tsui, H.L. Störmer, and A.C. Gossard, Phys. Rev. Lett. 48, 1559 (1982); H.L. Störmer, Physica B 177, 401 (1992).

[2] R.B. Laughlin, Phys. Rev. Lett. 50, 1395 (1983).

[3] T. Chakraborty and P. Pietiläinen, The Quantum Hall Effects (Springer, New York, 1995), second edition; T. Chakraborty, in Handbook on Semiconductors, vol. 1, ed. P.T. Landsberg (North-Holland, Amsterdam, 1992), Ch. 17.

[4] B.I. Halperin, Helv. Phys. Acta 56, 75 (1983).

[5] Theory: T. Chakraborty, Surf. Sci. 229, 16 (1990); T. Chakraborty, P. Pietiläinen, and F.C. Zhang, Phys. Rev. Lett. 57, 130 (1986); T. Chakraborty and F.C. Zhang, Phys. Rev. B 29, 7032 (1984); ibid. 30, 7320 (1984); P.A. Maksym, J. Phys. Condens. Matter 1, L6299 (1989).

Experiment: R.G. Clark et al., Phys. Rev. Lett. 62, 1536 (1989); J.P. Eisenstein, H.L. Störmer, L. Pfeiffer, and K.W. West, ibid., 1540 (1989); A.G. Davis et al., Phys. Rev. B 44, 13128 (1991); L.W. Engel et al., ibid 45, 3418 (1992); T. Sajoto, et al., ibid 41, 8449 (1990).

[6] Observation of fractionally charged quasiparticles is a direct confirmation of one of Laughlin's original predictions in [2]. See e.g., L. Saminadayar, D.C. Glattli, Y. Jin, and B. Etienne, Phys. Rev. Lett. 79, 2526 (1997).

[7] T. Chakraborty and P. Pietiläinen, Phys. Rev. Lett. 76, 4018 (1996); T. Chakraborty, P. Pietiläinen and R. Shankar, Europhys. Lett. 38, 141 (1997).

[8] I.V. Kukushkin, K. von Klitzing, and K. Eberl, Phys. Rev. 55, 10607 (1997).

[9] F.C. Zhang and T. Chakraborty, Phys. Rev. B 34, 7076 (1986).

[10] S.E. Barrett, et al., Phys. Rev. Lett. 74, 5112 (1995); E.H. Aifer, et al., ibid 76, 680 (1996).

[11] S.I. Dorozhkin, M.O. Dorokhova, R.J. Haug, and K. Ploog, Phys. Rev. B 55, 4089 (1997).

[12] T. Chakraborty, K. Niemelä, and P. Pietiläinen, Phys. Rev. Lett. 78, 4829 (1997).

[13] D. Yoshioka, Physica B 184, 86 (1993); J. Phys. Soc. Jpn. 62, 839 (1993); S. Tokizaki and Y. Kuramoto, J. Phys. Soc. Jpn. 64, 2302 (1995).

[14] B.I. Halperin, in Perspectives in Quantum Hall Effects, edited by S. Das Sarma and A. Pinczuk (Wiley, 1997), ch. 6; B.I. Halperin, P.A. Lee, and N. Read, Phys. Rev. B 47, 7312 (1993).

[15] W. Kang, H.L. Stormer, L.N. Pfeiffer, K.W. Baldwin, and K.W. West, Phys. Rev. Lett. 71, 3850 (1993). 
[16] D. Weiss, et al., Surf. Sci. 305, 408 (1994); I.V. Kukushkin, et al., Phys. Rev. Lett. 79, 1722 (1997).

[17] T. Chakraborty and P. Pietiläinen, Phys. Rev. B 53, 4664 (1996).

[18] G. Timp, R. Behringer, J.E. Cunningham, and R.E. Howard, Phys. Rev. Lett. 63, 2268 (1989); G. Timp, in Nanostructured Systems, ed. M. Reed (Academic, Boston, 1992), Ch. 3. 